\title{
EL POSTOTALITARISMO CARISMÁTICO Y EL CAMBIO DE RÉGIMEN: CUBA Y ESPAÑA EN PERSPECTIVA COMPRADAD
}

\author{
Eusebio Mujal-León y Jorge Saavedra *
}

\section{INTRODUCCIÓN}

“¿Qué hacer?” Esta pregunta, que alguna vez se planteara Lenin, resume hoy la problemática del establecimiento de la democracia en Cuba. ¿Qué hacer para ayudar al establecimiento de la democracia en Cuba? La cuestión es extremadamente compleja. No existen, ni creemos tener, respuestas simples o soluciones satisfactorias. En las siguientes páginas intentamos arrojar algunas luces sobre la incierta cuestión de la transición en Cuba desde la perspectiva de la política comparada.

Aunque nuestro análisis es motivado por el deseo de una pronta instauración democrática en Cuba, hemos resistido la tentación de dar por descontado que la única alternativa posible, o incluso la más probable, al régimen cubano actual, sea una democracia. En general, entonces, las preguntas a responder son las siguientes: ¿Qué va a ocurrir en Cuba? ¿Habrá una transición política? Si la hay, ¿consistirá más probablemente en una transición a la democracia o a algún tipo de régimen autocrático?

Evidentemente estas preguntas escapan por mucho a nuestras capacidades. De hecho la ciencia política no está aún preparada para predecir cambios políticos de esta naturaleza. Y nosotros, en ningún caso, pretendemos ser capaces de adivinar el futuro. Los análisis políticos retrospetivos resultan relativamente fáciles de hacer. Puestos a explicar el pasado "explicamos" coherente y convincentemente lo que en su momento era incoherente e impredecible. Pero esto no es prueba de una incipiente capacidad de la politología para predecir los cambios políticos. En cambio es un ejemplo de lo que Max Weber llamó "determinismo retrospectivo". En efecto, enfrentados a la necesidad de pronosticar, nos encontramos en una posición muy débil. En este sentido, el inesperado derrumbe de la Unión Soviética y de sus satélites en la Europa Oriental son buenos ejemplos de las limitaciones predictivas de la ciencia política.

De cualquier modo, en las siguientes páginas, y arriesgando un fracaso rotundo, intentaremos aportar algunas ideas sobre la posible evolución política en Cuba. El punto de partida de nuestro análisis es el régimen cubano de los noventa. Sólo si comprendemos adecuadamente el régimen cubano de la posguerra fría, y nos ayudamos comparando su situación actual con la de otros casos similares, podremos, tal vez, arrojar alguna luz sobre la cuestión del establecimiento de la democracia en Cuba. En efecto, el tipo de régimen es fundamental para comprender las perspectivas de su evolución en general y de su potencial evolución democrática en particular. A modo de ejemplo (y el lector haría bien en retener este ejemplo en su memoria), la relativamente fluida transición democrática española de los años setenta parece impensable, y sin duda hubiera sido infinitamente más difícil, desde un régimen como el franquista de los años cuarenta. Una caracterización del régimen cubano actual nos permitirá conocer sus bases de apoyo, sus principales fortalezas y debilidades, y las necesarias transformaciones que lo distancian de un régimen democrático. Dedicamos, en consecuencia, una buena parte de nuestro esfuerzo a la cuestión del tipo de régimen.

Además del tipo de régimen, para intentar atisbar las posibilidades de evolución del régimen cubano, creemos útil estudiar las condiciones internas y externas que a la vez constriñen y potencian dicha evolución. Para ello nos ayudamos del estudio comparado de otros regímenes que en mayor o menor medida estuvieron sujetos a las mismas condiciones que la Cuba actual (como China y Vietnam por ejemplo). En particular nos concentramos en el estudio del caso español, el que, aunque pueda sorprender a muchos, se compara muy bien con Cuba y nos permite extraer interesantes lecciones.

A continuación, entonces, intentamos caracterizar el régimen cubano desde la política comparada. Esta tarea nos obliga a realizar, por momentos, un análisis académico algo árido. Esperamos contar con la comprensión del lec-

Georgetown University. 
tor. Tenemos la esperanza de que lo que se pierda en fluidez y amenidad será más que compensado por una mejor comprensión del complejo fenómeno de la posible transición a la democracia en Cuba.

\section{EL TIPO DE RÉGIMEN CUBANO: POSTOTALITARISMO-CARISMÁTICO}

Juan Linz propuso en 1996 una nueva tipología de regímenes no democráticos, de gran utilidad para el estudio comparado de los procesos de democratización ${ }^{1}$. Linz distingue cuatro tipos de regímenes no democráticos: totalitario, postotalitario, autoritario y sultanista. Los tres primeros, junto con la democracia (representativa) o "poliarquía”, son regímenes contemporáneos par excellence. El sultanismo, en cambio, es un tipo tradicional, que opera sobre la base de la sola discrecionalidad del "sultán" o líder, sirviendo simplemente sus intereses personales.

Respecto de los tipos propiamente contemporáneos, esto es, totalitario, postotalitario, autoritario y democrático, existe una tendencia a considerarlos parte de una “escala horizontal continua". Pero esta metáfora suele despistarnos. En efecto, nos hace percibir esos regímenes como formas fluidas en vez de tipos distintos y, en consecuencia, nos lleva a suponer que cada régimen tiende a transformarse natural y gradualmente en su forma contigua. Aunque es cierto que en general el más probable cambio de régimen ocurre desde cada tipo a su forma adyacente, ello no es una necesidad. Así, por ejemplo, el paso desde el postotalitarismo a la democracia es perfectamente posible; fenómeno que observamos hoy en algunos países de la Europa oriental. El cambio de régimen tampoco es automático o necesariamente fluido; lo que no significa que los tipos sean estáticos. Cada categoría es un tipo único, con sus propias especificidades y lógica interna, lo que hace a cada régimen cualitativamente, y no sólo cuantitativamente, distinto de los demás. Esto, siendo cierto para los tipos teóricos, es aún más verdadero en el caso de los regímenes "de carne y hueso". Estos poseen características únicas que escapan a todo esfuerzo tipológico, pero que son fundamentales para comprender su evolución.

Es necesario tener también presente que todo régimen consiste en una forma de "ordenamiento" de las relaciones entre estado y sociedad. En consecuencia, todo régimen presupone un "equilibrio". Este punto nos permite distinguir la verdadera existencia de un tipo de régimen, de una situación meramente transitoria o de transformación de un régimen en otro. Este movimiento puede implicar simultáneamente la desintegración y la construcción de un nuevo tipo, pero no representa un régimen en sí mismo. Pero esto no significa que no pueda haber regímenes híbridos. Los regímenes híbridos no se ajustan a los tipos mencionados, pero poseen una especificidad y lógica interna propias. De hecho, la Cuba de los noventa es un híbrido, que hemos bautizado como "postotalitarismo-carismático", pues com- bina elementos típicamentemente postotalitarios con una exacerbación del elemento carismático típico de regímenes totalitarios. Esta particular combinación le da al régimen cubano de la posguerra fría unas tendencias muy particulares; y creemos que útiles para comprender las perspectivas para la democracia en Cuba.

¿Cómo caracterizar al régimen de Fidel en los años noventa? Como ya dijimos, se trata de un complejo híbrido que denominamos postotalitarismo-carismático. En general se caracteriza por el predominio de rasgos postotalitarios (o totalitarios "atenuados"), junto con una exacerbación del elemento carismático, que le da al régimen su carácter único; y una probada capacidad para adaptarse a un contexto sumamente adverso.

Un régimen totalitario se caracteriza por un "total control" del estado sobre la sociedad y, en consecuencia, por la ausencia de todo pluralismo, sea este político, económico o social. El estado, representado por un partido único, impone un único proyecto o interés colectivo. Este proyecto se manifiesta en una ideología totalitaria (utopía), en nombre de la cual la sociedad es intensa y extensamente movilizada. El liderazgo es típicamente carismático; stalinismo y nazismo son dos ejemplos típicos de este tipo de régimen.

En el caso de Cuba se aprecian los siguientes rasgos postotalitarios: a) Un naciente pluralismo económico, que se manifiesta en una calculada penetración del mercado en ciertos sectores de la economía, bajo los auspicios del estado cubano. Básicamentese trata de espacios abiertos a la inversión extranjera y a la economía familiar; además del surgimiento de una economía paralela, basada exclusivamente en el dólar. La economía cubana sigue estando bajo el casi completo control del estado. b) Un mínimo aunque incipiente pluralismo social, el que se observa básicamente en una creciente "autonomía" de las organizaciones religiosas; en particular de la Iglesia Católica. En todo caso, la sociedad civil y la oposición interna organizada siguen siendo, para todos los efectos prácticos, casi inexistentes. c) Un partido comunista debilitado y una ideología socialista deteriorada, probablemente en vías de extinción. Todo indica que en Cuba la utopía ya fue "desarmada". Se empieza a desnudar un núcleo ideológico nacionalista y antiamericanista. Aparentemente una racionalidad económica de mercado empieza a notarse en la isla. Sin embargo, no se malentienda, el estado mantiene un alto grado de control sobre la sociedad cubana. d) Como consecuencia de lo anterior, se observa una reducción en las capacidades y esfuerzos movilizadores por parte del régimen. Los valores de la sociedad cubana comienzan a "privatizarse".

1. Juan J. Linz y Alfred Stepan, Problems of Democratic Transition and Consolidation (Baltimore: The Johns Hopkins University Press, 1996).

2. La tercera ola es el título con el que Samuel Huntington bautizara la sorprendente marea mundial de democratizaciones, iniciada en Portugal en el mes de abril de 1974 y que se ha extendido hasta los años noventa. Samuel Huntington, La Tercera Ola (Barcelona: Paidós, 1991). 
El incipiente pluralismo económico y social debería jugar un papel central en una potencial democratización del régimen cubano. Es aquí donde una oposición interna puede finalmente florecer y provocar una dinámica de cambio de régimen. El amparo de la Iglesia Católica, por ejemplo, probó ser fundamental para el desarrollo y fortalecimiento de la sociedad civil en general y de la oposición política en particular durante "la tercera ola" 2; Chile y Polonia son dos buenos ejemplos. Recordemos que una distribución de fuerzas internas favorable a la democratización ha sido clave para explicar transiciones exitosas.

El elemento que, en conjunto con sus rasgos postotalitarios, le da su carácter específico al régimen cubano es la exacerbación del elemento carismático. Max Weber define el carisma como una importante fuente de legitimidad El líder carismático, según Weber, es obedecido en base a una confianza personal en sus cualidades excepcionales o ejemplares, en virtud de las cuales él o ella goza de poderes extraordinarios. En Cuba, el carisma de Fidel Castro se ha transformado en una de las bases principales, si no la principal, de sustentación del régimen; se refleja por ejemplo en la radical consigna "con Fidel todo, sin Fidel nada". Esta exacerbación del carisma resulta lógicamente de la descomposición de las principales fuentes de legitimidad del régimen, esto es, agotamiento ideológico, grave deterioro económico, social y moral, y un contexto internacional muy desfavorable. En otras palabras, la atenuación de los elementos totalitarios del régimen han aumentado decisivamente el peso relativo del carisma en relación a otras fuentes alternativas de legitimidad.

Vale la pena destacar dos elementos especialmente interesantes del poder carismático. Primero, su capacidad "revolucionaria", destacada por Weber. El líder carismático es por definición un innovador. Este elemento le da capacidad de innovacíon al régimen a través del líder, cuyo poder literalmente "extra-ordinario" le transforma en un actor político capaz de romper con "las escrituras" o ortodoxia del régimen. En este sentido es en buena medida el liderazgo de Castro lo que le otorga al actual régimen cubano su capacidad para adaptarse con éxito al nuevo y difícil entorno. Ello evidentemente supone el apoyo de los sectores claves del régimen, en particular, en este caso, de las fuerzas armadas.

Segundo, dado que el carisma descansa en cualidades, por definición la existencia de un régimen carismático corresponde, en el mejor de los casos, al "ciclo vital" del líder. La muerte o caída del líder significa el colapso del régimen a menos que el carisma sea "rutinizado" o institucionalizado de manera de preservar la posición dominante de las élites del régimen. Es esto lo que posiblemente intentarán hacer dichas élites, probablemente apostando por la que parece ser la salida más fácil: un régimen autoritario capitalista. Pero tal reforma es un arma de doble filo: por una parte permitiría la salvación del regimen, pero por otra supone el inicio de un duro proceso de reconversión y transformación económica, que no garantiza la persistencia del régimen dadas las condiciones actuales.

Finalmente, un importante grado de clientelismo caracteriza al régimen cubano de los noventa; en este sentido comparable quizás a México. El cientelismo se puede definir como unas relaciones desiguales de intercambio entre un patrón y un subordinado, de las cuales ambos se benefician social y económicamente. De alguna manera, la idea de "si tú me das, yo te doy" se institucionaliza, generando una fuerte resistencia al cambio social y político. Dada la relación clientelista, tiende a no haber incentivos individuales para abandonarla. El estado cubano, como el estado mexicano, es un "gran patrón", que garantiza el "bienestar" de la sociedad cubana. Este orden de cosas, sin embargo, es altamente vulnerable a una pérdida de capacidad por parte del patrón.

\section{CUBA Y ESPAÑA EN PERSPECTIVA COMPARADA}

En la sección anterior hemos definido el régimen cubano de la posguerra fría. Ello arroja luces sobre las perspectivas de cambio de régimen en Cuba. Sin embargo, para intentar comprender esta posible evolución debemos, al menos, conocer las principales condiciones internas y externas que constriñen el cambio. Las condiciones actuales de Cuba son las siguientes: a) Pérdida de capacidad por parte del estado y un pobre desempeño económico, principalmente debido al término del subsidio soviético. b) Un pueblo "cansado", más aún "desesperado". c) Existencia de un exilio importante, pero dividido. d) Situación internacional muy adversa; estado paria y asediado). e) Embargo comercial estadounidense.

$\mathrm{Si}$ podemos comparar el caso cubano con alguna experiencia similar, ello tal vez nos permitiría poner en perspectiva algunos de los principales problemas que enfrenta el régimen y, con suerte, algunas de sus posibles alternativas de desarrollo futuro. ¿Con qué podemos comparar el caso cubano? Necesitamos apoyarnos en el estudio comparado de otros regímenes similares y que, además, en mayor o menor medida hayan estado sujetos a las mismas condiciones que la Cuba de los años noventa. China y Vietnam son dos casos sin duda relevantes. En lo siguiente, sin embargo, nos concentraremos principalmente en el estudio del caso español, el que permite extraer importantes lecciones para Cuba.

Descartaremos desde un comienzo las experiencias de la Unión Soviética, América Latina y Europa oriental. Por distintas razones no son especialmente relevantes para explicar Cuba. La ruptura en la ex Unión Soviética (gobernada por una gerontocracia que ya no creía ni en sus propias palabras) se explica básicamente por la división al interior de la élite y el decisivo liderazgo de Gorbachev; además del enorme problema étnico nacional. Fidel Castro, en reciente entrevista, declaró: "Gorbachev hundió su barco. Estamos haciendo lo contrario de lo que él hizo”. 
En cuanto a América Latina, se trata de regímenes esencialmente diferentes, con una sociedad civil relativamente bien articulada y economías capitalistas; tal vez sólo el pinochetismo en su momento más álgido sea comparable, con reservas, a la situación cubana. Europa oriental tampoco es muy relevante ya que se trataba de países ocupados (la ex Yugoslavia es la principal excepción). Aquí, y en claro contraste con Cuba, los movimientos nacionalistas europeos fueron la base para la resistencia contra los regímenes comunistas, que carecían de verdadera legitimidad interna. Una lección sí podemos extraer de Europa oriental: la oposición interna al régimen probó ser fundamental para generar una transición a la democracia exitosa. Traducido al caso cubano esto significa que es una buena política todo aquello que ayude a fortalecer a los individuos y organizaciones que componen la oposición democrática interna a Fidel Castro.

Experiencias útiles son las de China y Vietnam. Ambos regímenes, en general bastante similares al cubano, se han movido exitosamente desde una posición comparable a la cubana hacia una economía cada vez más libre, sin ceder el estado su férreo control político sobre la sociedad. Dos de las claves del éxito de China y Vietnam han sido la unidad de la élite dirigente y el éxito económico de sus reformas. De hecho, Castro ha estado observando atentamente dichos casos. Recientemente declaró en una entrevista que "debemos adaptarnos a los nuevos tiempos; lo que no significa que debamos abandonar nuestros ideales. Adaptaremos el socialismo a una nueva realidad de una manera ordenada. Estamos mirando al modelo chino y vietnamita, y no al ruso". Sin embargo, existe una diferencia fundamental entre dichos regímenes y Cuba: ellos no están sometidos a la presión política y económica internacional que sufre la isla. China puede darse el lujo de experimentar.

Una comparación con el caso de España es particularmente interesante. La transición española de los setenta no es comparable con Cuba. No sólo porque Fidel aún vive y la transición española ocurrió sólo tras la muerte de Franco, sino además por otras importantes razones. En España la transición ocurrió desde un régimen autoritario, con una sólida economía de mercado y una desarrollada sociedad civil. Ninguna de estas son características de la Cuba actual. Además, durante los setenta la falange ya no existía como partido único y los militares estaban en pleno retroceso interno y no formaban ya parte del núcleo significativo del régimen. Hoy en Cuba el partido comunista es débil, más aún incapaz, y los militares son relativamente fuertes. Estos elementos son el resultado de las actuales características postotalitarias del régimen, las que resaltan la importancia de los militares, al mismo tiempo que el carisma del líder.

¿Cómo, entonces, comparar España y Cuba? Un momento en particular de la historia española contemporánea se asemeja notablemente a la situación cubana actual y este es el "primer franquismo", el régimen franquista de los años cuarenta; más precisamente entre 1939 y 1951.
Aquél fue también un caso de régimen autocrático asediado por una situación de aislamiento internacional y de grave desintegración interna. Es cierto que el primer franquismo fue, a diferencia de Cuba, probablemente un caso de autoritarismo.

Sin embargo la España de los cuarenta presenta notables semejanzas con un régimen totalitario: a) Total control político de la sociedad por parte del Estado. b) Partido único con ideología fascista. c) Tendencias fuertemente mobilizadoras coexistiendo con una feroz represión interna. Más interesante aún es el hecho de que, tal como en Cuba hoy, hacia fines de los cuarenta se observaba en España un creciente debilitamiento de los rasgos totalitarios del régimen; en particular de su contenido ideológico, en este caso fascista.

Pero las similitudes entre el caso español y cubano no acaban aquí. España, además, presentaba los siguientes rasgos: a) Un pueblo "cansado", producto de la guerra civil. b) Situación de miseria económica y social. c) Un exilio importante y dividido. d) Modelo económico esencialmente autárquico. e) Un líder con fuerte capacidad de liderazgo interno. f) Una situación internacional crecientemente adversa, que culminó en el aislamiento político y comercial del régimen español tras la derrota de Hitler. España para fines de los cuarenta, como la Cuba actual, era un "país paria" en el contexto occidental. Franco, como Fidel, llegó al poder por la fuerza de las armas, imponiéndose por medio de la represión y el exilio. Franco entonces, como Fidel hoy, se encontró en una situación de completa desubicación política e ideológica tras la derrota de sus aliado totalitario. Franco sobrevivió. ¿Qué hay de Fidel?

¿Qué nos dice el caso español respecto de las perspectivas de cambio de régimen en Cuba? ¿Cómo sobrevivió Franco? La resolución de 1946 de la Asamblea General de la ONU parecía anunciar la muerte del régimen franquista: "En origen, naturaleza, estructura y conducta general, el régimen de Franco es un régimen fascista... Evidencia documentada e incontrovertible establece que Franco fue parte culpable, junto con Hitler y Mussolini, en la conspiración para librar la guerra contra los gobiernos aliados". Menos de diez años después, en 1955, una España trinfante en la arena internacional sería admitida como miembro pleno a la ONU. ¿Cómo rompió Franco la situación de paria internacional?

Franco recurrió a una doble estrategia. Por una parte, alejó al régimen de su identidad fascista, reciclándose a través del desarrollo de una nueva identidad nacional católica. El acercamiento con el Vaticano, además de expandir su legitimidad interna, le permitió al régimen contar con un poderoso aliado en la arena internacional. Esta estrategia fue reforzada por la creación de un nuevo régimen jurídico que al menos en el papel reconocía le existencia de ciertas libertades individuales básicas, iniciando un movimiento hacia un estado de derecho autoritario. Por otra parte, Franco enfatizó el carácter anticomunista del régimen español, buscando siempre transformarse en un aliado de 
los Estados Unidos. Esta última sería finalmente la clave del éxito y el régimen se salva gracias a la nueva situación internacional de posguerra.

1949, el año del lanzamiento de la Guerra Fría, fue un excelente año para Franco. En abril se crea la OTAN, en julio el papa excomulga a "comunistas e izquierdistas" y, el mismo mes, la Unión Soviética detona su primera bomba atómica. En 1950 la oposición interna española (dividida en el exilio) se encontraba totalmente derrotada y los militares estaban bajo el sólido control del régimen. España pasaba ahora la ofensiva. 1950 fue el año de Franco. Este año la Asamblea General de la ONU anuló la resolución del año 46. La Guerra de Corea le dio a Franco la oportunidad de luchar junto a Estados Unidos. El régimen franquista había sobrevivido.

En suma, el franquismo se recicla en torno al nacional catolicismo y se gana a las potencias occidentales, particularmente a los Estados Unidos, gracias a su agresivo anticomunismo. El mundo de la Guerra Fría proveyó a Franco con una oportunidad que fue bien aprovechada por el caudillo español. ¿Proporciona el mundo de la posguerra fría una oportunidad similar a Fidel?

Así como la Guerra Fría y una astuta política exterior proporcionaron un importante sustento al regimen anticomunista de Franco, no es claro que el fin de dicha "guerra ideológica” vaya a derribar a Fidel. De hecho, paradójicamente, en alguna medida podría ayudar a las fuerzas antidemocráticas cubanas; en parte reforzando la unidad de las élites frente a la amenaza externa o, en parte también reforzando el carisma del líder entendido como la "última esperanza”. De cualquier modo, la experiencia española parece indicar que si esas élites desean permanecer en el poder en un contexto internacional tan desfavorable como el actual, el régimen debe evolucionar. En términos ideológicos, sin embargo, sus posibilidades de evolución parecen ser limitadas. Al menos bajo el liderazgo de Fidel Castro.

Franco pudo adaptarse, mirado retrospectivamente, con relativa facilidad, porque su sistema económico no estaba en contradicción con el sistema económico capitalista de las potencias vencedoras. España pudo y supo ganarse a los Estados Unidos, la potencia occidental internacional. La adaptación fue meramente política: alineamiento ideológico y evolución hacia un "autoritarismo moderado", un régimen que probó ser "aceptable" para sus vecinos europeos. Cuba, en cambio, requiere de un cambio económico mucho más profundo y, por lo tanto, mucho más difícil. Además, las posibilidades de un acercamiento cubano-americano bajo Fidel se ven bastante más difíciles; aunque probablemente se veían igualmente difíciles en la España de los cuarenta. Por último, hoy en occidente los estándares de "aceptabilidad" de un régimen político suponen unas condiciones mucho mayores. Un "autoritarismo moderado" (que en todo caso no el caso de Cuba) ya no es suficiente; por lo menos se requieren elecciones libres.

Tal vez la mayor limitante proviene del carácter del régimen cubano, que a nuestro juicio dificulta una transi- ción hacia una plena economía de mercado. Un régimen postotalitario-carismático tiene una lógica opuesta a la del mercado. No sólo la lógica del mercado entra en conflicto con el postotalitarismo, sino que además se contradice con el carisma del líder. El carisma supone, por definición, un grado de arbitrariedad que hace a los actores económicos vulnerables a las políticas del régimen. Parece ser que bajo estas circunstancias será muy difícil que Cuba vaya más allá de garantizar "enclaves capitalistas", o una "economía paralela oficial”; sea en ciertos sectores particulares de la economía (turismo, por ejemplo) o en ciertas áreas geográficas (como ocurre actualmente en China).

El carisma es un arma de doble filo. Por una parte es una "fuerza revolucionaria" y que por lo tanto otorga flexibilidad. Sólo el líder carismático es capaz de romper con la "ortodoxia". En este sentido, el rol de Castro parece haberle dado al régimen cubano una particular capacidad de adaptación. Pero, por otra parte, Fidel representa los valores del régimen: socialismo, nacionalismo y antiamericanismo. ¿Hasta adónde puede Castro abrirse a las fuerzas del mercado? Mientras Fidel utilice las concesiones al libre mercado y al capital extranjero como instrumentos para la preservación de los "valores del régimen", estos es, socialismo (cada vez más venido a menos), nacionalismo y antiamericanismo, entonces tiene esperanza de mantenerse en el poder y preservar el régimen actual. Sin embargo, ello también significa que se le cierra la puerta a una reforma económica profunda que suponga finalmente una economía de mercado plena. En efecto, en las circunstancias actuales, la libre empresa seguirá siendo en Cuba algo excepcional, sujeta a la autorización del régimen.

Por último, existe una notable y fundamental diferencia entre el primer franquismo y el fidelismo tardío. En efecto, Franco se encontraba en su apogeo durante los años cuarenta; Fidel, en cambio, se encuentra en el ocaso de su carrera. La crisis de sucesión no era un problema de corto o mediano plazo para el franquismo, pero lo es sin duda para el actual régimen cubano. De hecho este problema nunca pudo ser resuelto por Franco y el régimen no sobrevivió su muerte. La enorme importancia de la figura de Fidel, bien establecida cuando discutimos el elemento carismático exacerbado que caracteriza a la Cuba actual, hace al régimen particularmente vulnerable a la cuestión de la sucesión. Dado el intenso componente carismático del régimen cubano, la muerte de Fidel, sin duda, acabará con el tipo de régimen que llamamos "postotalitarismo-carismático" (lo que no disminuye en nada su caracterizacíon como un tipo único y su utilidad para el estudio comparado). Al mismo tiempo, la muerte de Fidel es el suceso que con mayor probabilidad abrirá las puertas a una transición a la democracia en Cuba, o sea, "con Fidel todo, excepto democracia". Pero que no se nos malentienda: nada garantiza tal democratización. El proceso es muy complejo y no hay forma de saber qué tipo de dinámica generaría la muerte del líder. La transición a la democracia en Cuba no debe darse por sentada, como si fuera simplemente la "crónica de una muerte anunciada". 


\section{RESUMEN}

En el texto se intentan aportar algunas ideas sobre la posible evolución política en Cuba, partiendo del análisis del régimen cubano de los noventa, y comparando su situación con la de otros casos similares. En este sentido, definen el actual régimen cubano como postotalitarismo-carismático e intentan analizar las condiciones internas y externas que constriñen y potencian la evolución del régimen cubano.

Palabras claves: postotalitarismo-carismático, transición española.

\section{ABSTRACT}

This article analyses the prospects of the future Cuban political evolution. It does so by taking into account the characteristics of the Cuban regime during the 1990's and by comparing the Cuban situation to that of other similar cases. This article characterizes the current political regime as charismatic-post-totalitarianism. It also analyzes the internal and external factors that affect and constrain the evolution of the Cuban regime.

Key words: charismatic-post-totalitarianism, Spanish transition.

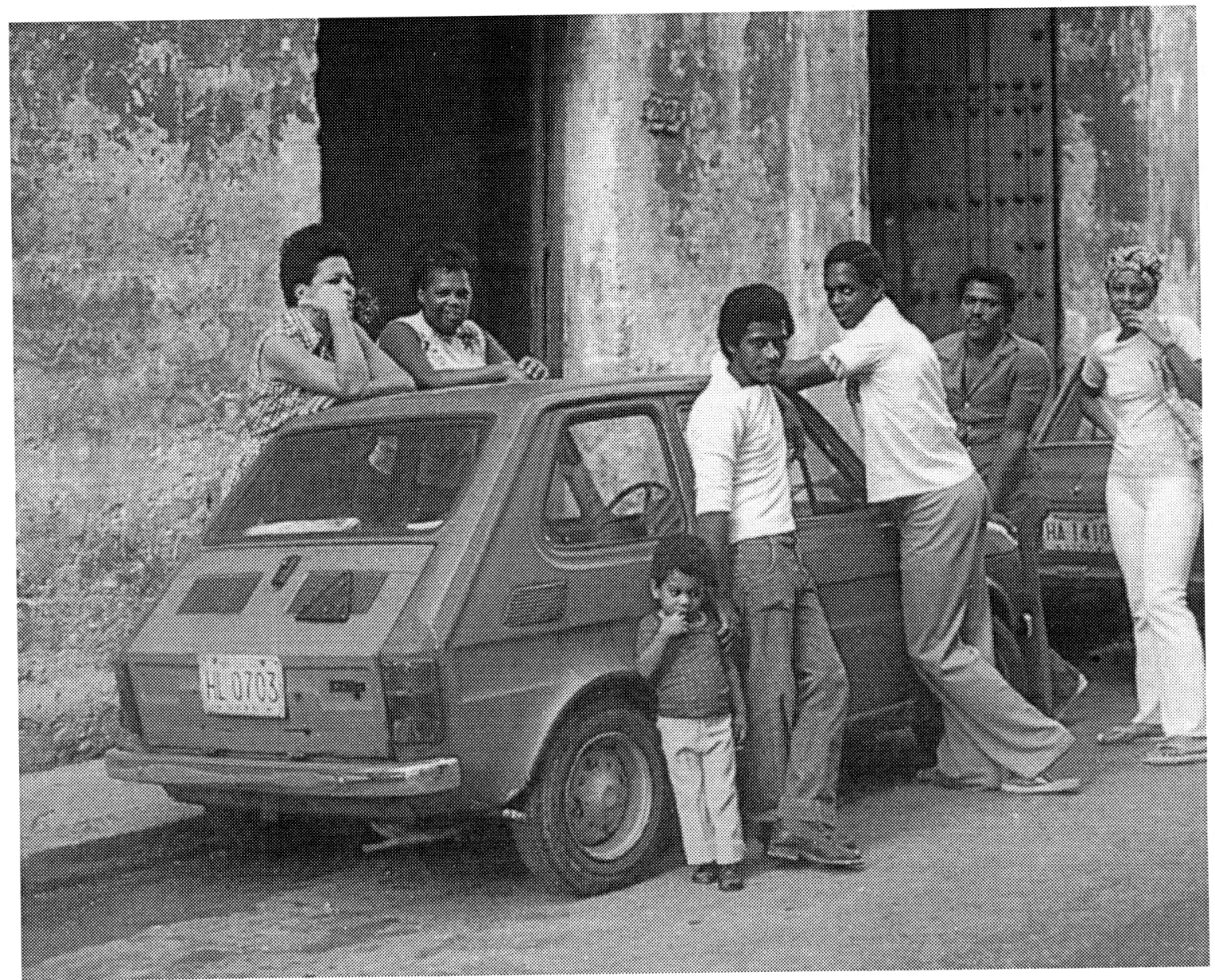

